\title{
Porphyromonas gingivalis Gingipains Selectively Reduce CD14 Expression, Leading to Macrophage Hyporesponsiveness to Bacterial Infection
}

\author{
Asaf Wilensky $^{a}$ Rinat Tzach-Nahman ${ }^{a, b}$ Jan Potempac, d Lior Shapira ${ }^{a}$ \\ Gabriel Nussbaum ${ }^{b}$ \\ ${ }^{a}$ Department of Periodontology and ${ }^{b}$ Institute of Dental Sciences, Faculty of Dental Medicine, Hadassah Medical \\ Center, Hebrew University, Jerusalem, Israel; ' ${ }^{C}$ Center of Oral Health and Systemic Disease, School of Dentistry, \\ University of Louisville, Louisville, Ky., USA; ${ }^{d}$ Department of Microbiology, Faculty of Biochemistry, Biophysics and \\ Biotechnology, Jagiellonian University, Krakow, Poland
}

\section{Key Words}

CD14 · Gingipains · Macrophages · Porphyromonas

gingivalis $\cdot$ Toll-like receptors $\cdot$ TNFa

\begin{abstract}
Cysteine proteases (gingipains) from Porphyromonas gingivalis are key virulence factors in chronic periodontitis. Innate immune receptors CD14, Toll-like receptor (TLR) 2 and TLR4 are important in $P$. gingivalis recognition. We examined the ability of gingipains to cleave CD14, TLR2 and TLR4, and the consequences for the cellular response to bacterial challenge. Macrophages were exposed to $\operatorname{Arg}$ (RgpA and RgpB)and Lys (Kgp)-gingipains, and residual expression of TLR2, TLR4 and CD14 was determined by flow cytometry. The cellular response to live bacteria following exposure to purified gingipains was evaluated by TNFa production and bacterial phagocytosis. RgpA and Kgp decreased CD14 detection in a concentration ( $p=0.0000002)$ - and time $(p=0.03)$-dependent manner, whereas RgpB had no significant effect. TLR2 and TLR4 expression were unaffected. Reduction in CD14 expression was more efficient with Lys-gingipain than with Arg-gingipain. A reduced CD14 surface level correlated with decreased TNFa secretion and bacterial phagocytosis following challenge with live $P$. gingivalis, but the response to heat-killed bacteria was unaffected. Therefore, gingipains
\end{abstract}

reduce CD14 expression without affecting expression of the bacterial-sensing TLRs. Reduced CD14 expression depends on the gingipain hemagglutinin/adhesion site and results in macrophage hyporesponsiveness to bacterial challenge. Further studies are needed to determine if reduced CD14 expression is linked to periodontitis induced by P. gingivalis.

(c) 2014 S. Karger AG, Basel

\section{Introduction}

Porphyromonas gingivalis is one of the major pathogens associated with periodontal disease [1]. Several virulence factors have been identified that enable $P$. gingivalis to induce chronic inflammation and alveolar bone loss in animal models of periodontal disease. These include fimbriae, lipopolysaccharides (LPSs), toxic metabolic products and proteases [2]. Two types of cysteine proteases, referred to as gingipains, account for $85 \%$ of the proteolytic activity of $P$. gingivalis. Rgp proteases are specific for arginine-Xaa bonds and Kgp protease is specific for lysine-Xaa bonds [3]. Two distinct but related genes encode

Asaf Wilensky, Rinat Tzach-Nahman, Lior Shapira and Gabriel Nussbaum contributed equally to this work.

\section{KARGER 125\%}

(c) 2014 S. Karger AG, Base

$1662-811 \mathrm{X} / 14 / 0072-0127 \$ 39.50 / 0$

E-Mail karger@karger.com

www.karger.com/jin
Dr. Gabriel Nussbaum

Institute of Dental Sciences, Faculty of Dental Medicine

Hadassah Medical Center, Hebrew University

Jerusalem (Israel)

E-Mail gabrieln@ekmd.huji.ac.il 
for Rgp proteases, $\operatorname{rgp} A$ and $\operatorname{rgpB}$. The proteins RgpA (95 $\mathrm{kDa})$ and $\mathrm{RgpB}(50 \mathrm{kDa})$ share a nearly identical catalytic domain (93\% sequence identity) but differ in that RgpB does not contain a hemagglutinin/adhesion domain. Both RgpA and Kgp are noncovalent complexes containing separate catalytic and hemagglutinin/adhesin domains. The catalytic subunits of RgpA and Kgp share 23\% identity whereas their hemagglutinin/adhesion domains are over $97 \%$ identical [4]. Gingipains cleave a wide range of proteins and thereby provide the bacteria with essential nutrients. In addition, gingipains interfere with the host recognition and response to infection by cleaving extracellular matrix proteins, antibacterial peptides, cytokines such as TNFa [5], cell surface receptors and immunoglobulins [6].

Toll-like receptors (TLRs) represent a conserved family of receptors involved in the detection of pathogen-associated molecular patterns (PAMPs) and the cellular response to bacterial invasion [7]. TLRs recognize bacterial PAMPs such as LPS recognized by TLR4 [8], lipopeptides recognized by TLR2 [9] and unmethylated CpG oligonucleotides recognized by TLR9 [10]. CD14 is a glycophosphatidyl inositol-anchored membrane protein that functions as a coreceptor for the recognition of bacterial molecules by several TLRs [11, 12]. Multiple bacterial pathogens evade innate immune responses by interfering with robust TLR-mediated recognition that would normally lead to rapid bacterial clearance [13, 14]. Several groups have found that TLR2 is required for a full cytokine response to infection with $P$. gingivalis $[15,16]$ raising the question of whether $P$. gingivalis evades clearance by reducing recognition through TLR2. Previously, gingipains were shown to reduce CD14 surface expression in human monocytes while not affecting TLR4 expression [17]. The aim of the present study was to examine the ability of Arg (RgpA, RgpB) and Lys (Kgp) gingipains to reduce the expression of the murine macrophage receptors TLR2, CD14 and TLR4, and to determine the effect of changes in receptor expression levels on the macrophage response to infection with live $P$. gingivalis.

\section{Materials and Methods}

\section{Animals}

Six- to 12 -week-old female BALB/c mice (Harlan, Jerusalem, Israel) were used. The study was carried out in the specific pathogen-free unit of the animal facility. The mice were maintained on a 12-hour light/dark cycle and received distilled water and food ad libitum. The experimental protocols were approved by the Internal Review Board of the Hadassah-Hebrew University Medical Center.

\section{Materials}

RAW 246.7 (ATCC) and $c d 14-/-$ murine macrophage (BEI Resources) cell lines were maintained in cell culture plates with DMEM supplemented with fetal calf serum (FCS; 10\%), $4 \mathrm{mM}$ L-glutamine, $1 \mathrm{~mm}$ sodium pyruvate and penicillin-streptomycin (1\%). Cells were used between passages 4-10. FITC (SigmaAldrich) was prepared in $\mathrm{NaHCO}_{3}$ buffer $(\mathrm{pH}=9)$ at $0.1 \mathrm{mg} / \mathrm{ml}$. Purified anti-mouse CD16/32, FITC-conjugated anti-mouse CD14 (Sa2-8), PE-conjugated anti-mouse TLR4 (MTS510), PEconjugated anti-mouse TLR2 (6C2) and isotype-matched controls were purchased from eBioscience (San Diego, Calif., USA) and used according to the manufacturer's instructions. A mouse TNFa ELISA MAX ${ }^{\mathrm{TM}}$ standard kit was purchased from Biolegend (San Diego, Calif., USA).

\section{Mouse Peritoneal Macrophage Isolation}

Each mouse was injected intraperitoneally with $2 \mathrm{ml} 4 \%$ thioglycollate (DIFCO) [18]. Four days later, mice were sacrificed and the peritoneal cavity was washed five times with $3 \mathrm{ml}$ PBS plus heparin ( 5 units/ml). Peritoneal exudate cells (PECs) were incubated in DMEM, $10 \%$ FCS, 4 mM L-glutamine, $1 \mathrm{mM}$ sodium pyruvate and penicillin-streptomycin (1\%). The following day, cells were harvested, washed with PBS and suspended in 0.2 M HEPES buffer containing $10 \mathrm{mM}$ cysteine and $5 \mathrm{mM} \mathrm{CaCl}_{2}$ at $37^{\circ} \mathrm{C}$ for $10 \mathrm{~min}$ (in order to activate gingipains).

\section{Bacteria}

$P$. gingivalis strain 381 (ATCC), W83 (wild type, WT), rgpAand $k g p$ - deficient strains were used in this study (W83, $r g p A-$ and $k g p$ - strains were kindly provided by C.A. Genco, Boston University, Boston, Mass., USA). Bacteria were grown in Wilkins media (Oxoid, Basingstoke, UK) under anaerobic conditions $\left(85 \% \mathrm{~N}_{2}\right.$, $5 \% \mathrm{H}_{2}$ and $10 \% \mathrm{CO}_{2}$ ) at $37^{\circ} \mathrm{C}$. After $2-3$ days, the bacteria were harvested and supernatants from each strain were collected and stored at $-80^{\circ} \mathrm{C}$ for further use. Protease activity of bacterial supernatants was tested as previously described $[19,20]$. To test phagocytosis, $P$. gingivalis 381 was labeled with $0.1 \mathrm{mg} / \mathrm{ml}$ FITC (Sigma-Aldrich) in $\mathrm{NaHCO}_{3}$ buffer (pH 9) for $20 \mathrm{~min}$ at room temperature, as previously described [16].

\section{Arg- and Lys-Gingipain Purification}

RgpB was purified from strain H66 culture fluid as previously described [21] using a combination of gel filtration and ion-exchange chromatography. Kgp and RgpA proteinase-adhesin complexes were isolated by the sequential use of gel filtration, arginineSepharose chromatography and anion exchange chromatography on Mono Q [22]. Purified protease activity was tested as previously described $[19,20]$.

\section{Macrophage Receptor Expression following Exposure to} Gingipains

Macrophages (PECs or RAW 264.7 cells) were incubated individually with culture supernatants from W83 WT, rgpA- or kgpP. gingivalis, or with purified RgpA, RgpB or Kgp at the concentrations and time periods indicated in the figures. After incubation, the cells were washed with $0.5 \mathrm{ml}$ FACS buffer (98\% PBS $+2 \%$ FCS), and receptor expression was determined by FACS. Macrophage $\mathrm{Fc}$ receptors were blocked with purified anti-mouse $\mathrm{CD} 16 / 32\left(4^{\circ} \mathrm{C}, 15 \mathrm{~min}\right)$ and cells were loaded with FITC/PE-conjugated monoclonal antibodies or isotype-matched rat IgG con- 
trols $\left(4^{\circ} \mathrm{C}, 45 \mathrm{~min}\right)$. Flow-cytometric analyses were performed using a BD LSRII (Becton Dickinson, Mountain View, Calif., USA). The mean fluorescence intensity (MFI) of viable cells was analyzed by gating on the basis of forward and side scatter characteristics. MFI results are shown as the mean \pm SEM for each group.

\section{Macrophage Stimulation}

In some experiments, macrophages were treated with $1 \mu \mathrm{M}$ of purified Kgp for $30 \mathrm{~min}$. Cells were washed three times in PBS containing 2\% FCS in order to inactivate the exogenously added gingipain prior to challenging them with live or heat-killed and sonicated (HKS) $P$. gingivalis at increasing multiplicity of infection (MOI), or with the purified TLR ligands Escherichia coli LPS (Sigma Aldrich, Rehovot, Israel), $P$. gingivalis LPS or $\mathrm{Pam}_{3} \mathrm{CSK}_{4}$ (Invivogen, San Diego, Calif., USA). Supernatants were collected and TNFa levels were determined by ELISA according to the manufacturer's instructions (mouse TNFa ELISA MAX ${ }^{\mathrm{TM}}$ standard; Biolegend). In addition, cells were stained with FITC-conjugated antimouse CD14 monoclonal antibody (or isotype control) in order to evaluate the level of detectable CD14 prior to and at the end of the 3-hour incubation with $P$. gingivalis. Cell viability at the end of the 3 -hour incubation was determined by propidium iodide staining.

\section{P. gingivalis Phagocytosis Assay}

Macrophages were pre-incubated with either purified RgpA or Kgp $(1 \mu \mathrm{M})$ for $30 \mathrm{~min}$. Cells were washed and incubated with FITC-labeled $P$. gingivalis (MOI 10) for 15-120 min. Extracellular labeling was quenched using trypan blue $(1.25 \mathrm{mg} / \mathrm{ml})$ for $1 \mathrm{~min}$ before determining fluorescence (Tecan fluorescence plate reader; Tecan, Männedorf, Switzerland).

\section{Statistical Analysis}

Experiments in this study were conducted at least twice. Experimental values are given as means \pm SEM of macrophage MFI for TLR2/TLR4/CD14 staining. In order to compare different exposure times and concentrations, we used the Kruskal-Wallis test. This was done for each innate immune receptor and each protease separately. Multiple pairing analyses were done with the Mann-Whitney and Bonferroni post hoc test for significance. To simultaneously estimate the effects of time and protease concentration, three-way ANOVA was used. Multiple paired comparisons were done according to Scheffé. This analysis was done for each innate immune receptor separately. TNFa secretion levels by stimulated macrophages were compared using Student's t test. All statistical analyses were two tailed and a value of 0.05 or less was considered statistically significant.

\section{Results}

\section{P. gingivalis Culture Supernatant Reduces CD14}

Surface Expression, but Not That of TLR2 or TLR4

Detectable macrophage CD14 surface expression was reduced following exposure to culture supernatants of W83, rgpA- and $k g p-P$. gingivalis at all concentrations tested ( $\mathrm{p}<0.01$; fig. 1a). However, although W83 supernatant was equally active at all concentrations tested, both $r g p A$ - and $k g p$ - supernatants were less active at the lowest concentrations tested, suggesting that both gingipains are required for optimal activity. Nevertheless, CD14 levels were least affected by the $k g p$ - strain, suggesting that Kgp is responsible for more of the activity than RgpA (fig. 1a, d). Following treatment with the W83, $r g p A-$ and $k g p-$ supernatants, the surface expression of TLR4 (fig. 1b) and TLR2 (fig. 1c) remained unchanged at even the highest concentration. Taken together, these results suggest that both Arg- and Lys-gingipains are able to specifically cleave CD14, but neither gingipain nor other $P$. gingivalis proteases affect macrophage TLR4 or TLR2 expression.

\section{TLR2, TLR4 and CD14 Surface Expression following} Macrophage Exposure to Purified Gingipains

We next tested the dose- and time-dependent effect of purified gingipains on the expression of CD14, TLR4 and TLR2 on murine macrophages. Overall, purified gingipains RgpA and Kgp reduce CD14 expression in a time-, concentration- and protease-type-dependent manner $(\mathrm{p}<0.05)$. At the shortest time period tested $(5 \mathrm{~min}$ exposure followed by washing), and the two lowest concentrations of gingipain $(0.1$ and $0.3 \mu \mathrm{M})$, only Lys-gingipain (Kgp) significantly reduced CD14 expression (fig. 2a), further suggesting that Kgp more efficiently reduces CD14 expression compared to Rgp. At higher concentrations of gingipain or longer exposure times, Arg-gingipain (RgpA) also significantly reduced CD14 surface expression (fig. 2a-c). Finally, the Rgp adhesin domain is required for efficient reduction in surface CD14, as RgpB, which lacks the adhesin domain, was inactive except for a modest effect at the longest exposure time $(1 \mathrm{~h})$ and highest concentration ( $1 \mu \mathrm{M}$; fig. $2 \mathrm{c})$. Similar to the culture supernatants, purified gingipains did not reduce surface-detectable expression of TLR2 or TLR4 (fig. 2d, e). For figures 1 and 2, comparable results were obtained using primary PECs and the murine macrophage cell line RAW 264.7.

\section{Kgp-Mediated Reduction of CD14 Expression}

Correlates with a Diminished Cytokine Response to

Infection with Live P. gingivalis

We next determined whether diminished surface expression of CD14, in the presence of unchanged levels of TLR2 and TLR4, would impact on the cytokine response to P. gingivalis. Macrophages were exposed to Kgp ( $1 \mu \mathrm{M}$ for $30 \mathrm{~min}$ ), washed three times in medium containing serum and then stimulated for $3 \mathrm{~h}$ with either live or HKS $P$. gingivalis, $P$. gingivalis LPS, E. coli LPS or the synthetic lipopeptide $\mathrm{Pam}_{3} \mathrm{CSK}_{4}$. Three hours following exposure to Kgp and washing, the CD14 level remained reduced by $60 \%$ compared to control cells not exposed to protease 


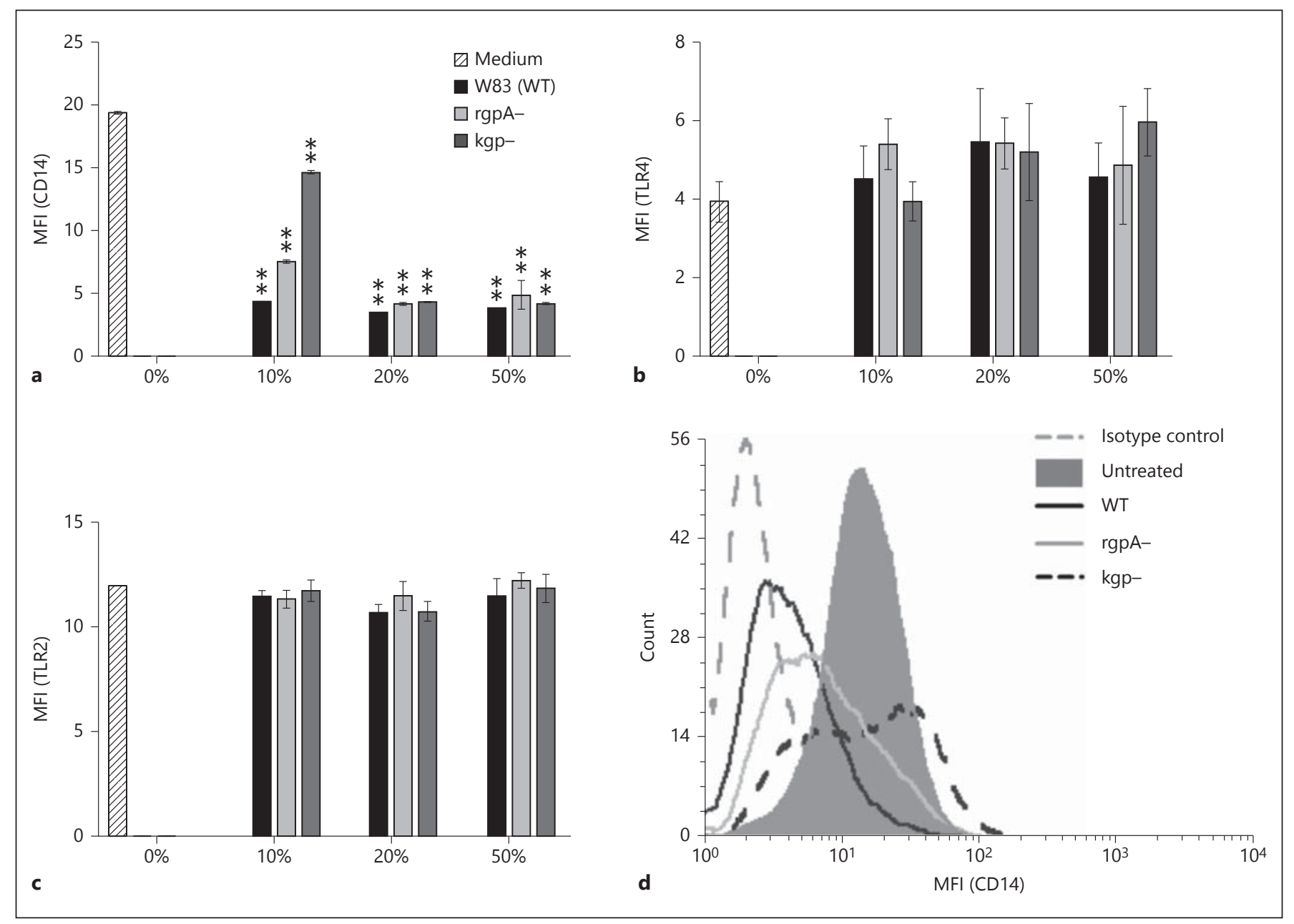

Fig. 1. $P$. gingivalis culture supernatant cleaves CD14 but not TLR2 or TLR4. CD14 (a), TLR4 (b) and TLR2 (c) surface expression detected following murine macrophage (PEC) exposure to W83 (WT), rgpA- and $k g p-P$. gingivalis culture supernatants. Culture supernatants were collected from bacteria at $6 \times 10^{6} \mathrm{CFU} / \mathrm{ml}$. PECs were incubated with supernatants at increasing concentrations $(10,20$ and $50 \%)$ or with Wilkins medium as a control. Following

(fig. 3a). In addition, macrophage viability was not affected by exposure to Kgp or the further 3-hour activation (data not shown). As shown in figure 3b, Kgp pretreatment led to a significant decrease in TNFa secretion by cells responding to infection with live $P$. gingivalis at MOIs of 10 and $50(\mathrm{p}<0.05)$, although the diminished response was not observed at MOI 100. The response to $P$. gingivalis LPS and E. coli LPS was also diminished by Kgp pretreatment $(\mathrm{p}<0.05)$. However, the response to HKS $P$. gingivalis, where additional PAMPs are presumably revealed, and the response to TLR2/1 activation by $\mathrm{Pam}_{3} \mathrm{CSK}_{4}$, was unaffected by Kgp pretreatment (fig. 3b). incubation for $1 \mathrm{~h}$, supernatants were discarded and macrophage CD14, TLR4 and TLR2 surface expression was detected using fluorescent antibodies. MFI was evaluated by flow cytometry. The values represent means \pm SEM. ${ }^{* *} \mathrm{p}<0.01$ vs. untreated. d Representative histogram of macrophage CD14 surface expression following exposure to $10 \%$ culture supernatant of WT, rgpA- or $k g p-$ $P$. gingivalis.
Similar results were obtained under serum-free conditions, suggesting that soluble bovine CD14 in the medium cannot compensate for the reduced membrane CD14 expression (data not shown). These results suggest that live $P$. gingivalis may dampen early cellular responses to infection by reducing surface CD14 levels.

\section{TNFa Secretion by WT or CD14-/- Macrophages} following Stimulation with Live P. gingivalis

To confirm that CD14 is specifically required for a full cytokine response to live $P$. gingivalis challenge, we compared TNFa production by WT versus $c d 14-/-$ macro- 

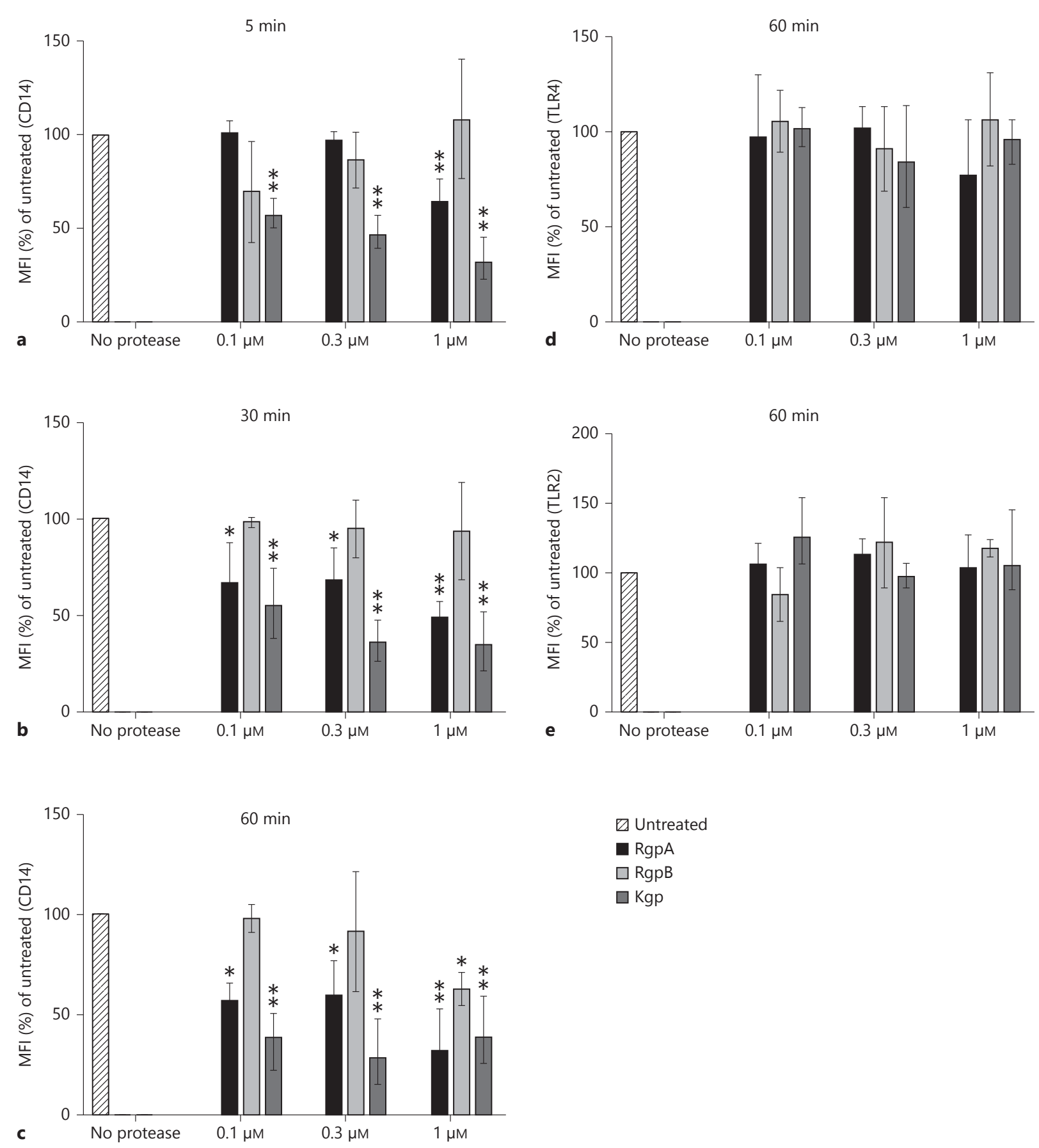

Fig. 2. Purified Kgp and RgpA selectively cleave CD14. Macrophage CD14 (a-c), TLR4 (d) and TLR2 (e) surface expression following PEC exposure to purified gingipains. Macrophages were treated with RgpA, RgpB and Kgp (0.1, 0.3 and $1 \mu \mathrm{M}$ for 5, 30 and $60 \mathrm{~min})$. CD14, TLR4 and TLR2 were detected using fluorescent antibodies following protease removal and MFI was evaluated by

flow cytometry. Results represent means of three independent experiments. The MFI of live-treated cells was compared to the MFI of viable untreated cells, which was set as $100 \%$. Cell viability was on average $65 \%$ and did not differ between protease-treated and untreated cells. ${ }^{*} \mathrm{p}<0.05$ vs. untreated cells. 


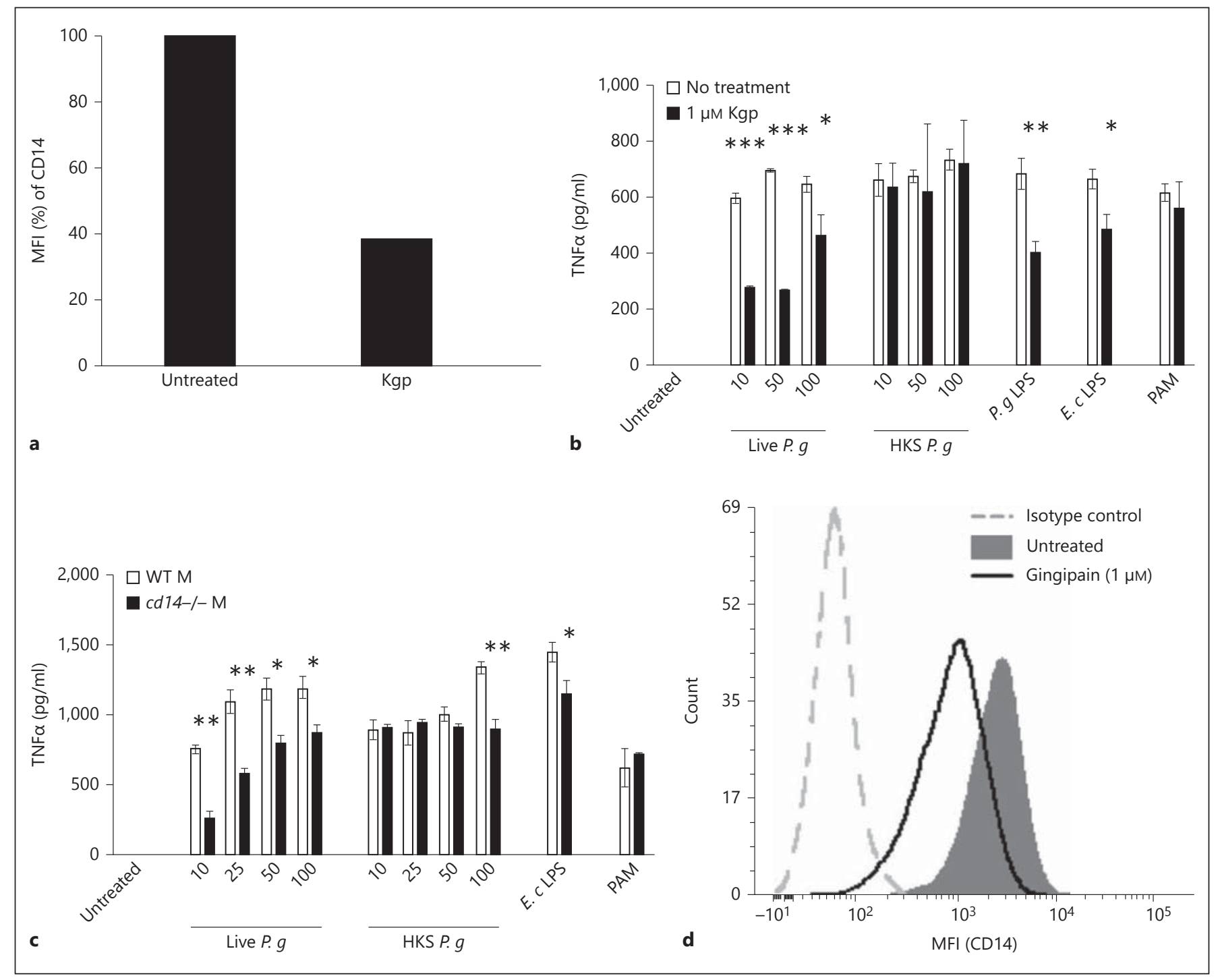

Fig. 3. Macrophages exposed to Kgp are hyporesponsive to live $P$. gingivalis (P. g). a CD14 surface expression (RAW 264.7 cells) following exposure to Kgp for $30 \mathrm{~min}$ and further $3 \mathrm{~h}$ of incubation with no stimulation. CD14 expression by cells not exposed to Kgp is defined as $100 \%$. b TNFa secretion by macrophages pretreated with Kgp and then activated. RAW 264.7 cells were treated with $1 \mu \mathrm{M}$ of Kgp for $30 \mathrm{~min}$. Cells were washed extensively and then challenged with live $P$. gingivalis or HKS $P$. gingivalis, (at $\mathrm{MOI}=$ 10,50 and 100), P. gingivalis LPS $(1 \mu \mathrm{g} / \mathrm{ml})$, E. coli (E. c) LPS $(10$ $\mathrm{ng} / \mathrm{ml}$ ) or $\mathrm{Pam}_{3} \mathrm{CSK}_{4}(\mathrm{PAM}, 10 \mu \mathrm{g} / \mathrm{ml})$ for $3 \mathrm{~h}$. Values represent

phages (fig. 3c). Consistent with the activity of Kgp in reducing surface CD14, macrophages that do not express CD14 are hyporesponsive to challenge with live P. gingivalis $(\mathrm{p}<0.001)$. In contrast to Kgp pretreatment, even at MOI $100 c d 14-/$ - cells secrete significantly less TNFa than WT cells $(\mathrm{p}<0.05)$, presumably since there is a com- means \pm SEM. ${ }^{*} \mathrm{p}<0.05,{ }^{* *} \mathrm{p}<0.001,{ }^{* * *} \mathrm{p}<0.0001$, vs. untreated cells. c TNFa secreted by WT (RAW 264.7 cells) or $c d 14-/-$ macrophages (M) following challenge. Macrophages were exposed to live $P$. gingivalis, HKS P. gingivalis, E. coli LPS $(10 \mathrm{ng} / \mathrm{ml})$ or $\mathrm{Pam}_{3} \mathrm{CSK}_{4}(10 \mu \mathrm{g} / \mathrm{ml})$ concentrations for $3 \mathrm{~h}$. TNFa secretion was determined by ELISA. Values represent means \pm SEM. ${ }^{*} \mathrm{p}<0.05$, ** $\mathrm{p}<0.001$, WT vs. $c d 14-/-$ macrophages. $\mathbf{d}$ Representative histogram of macrophage CD14 surface expression following exposure to $1 \mu \mathrm{M}$ gingipain.

plete absence of CD14 in this system. Importantly, the response to E. coli LPS was reduced in $c d 14-/-$ macrophages compared to WT, and the responses to HKS $P$. gingivalis and $\mathrm{Pam}_{3} \mathrm{CSK}_{4}$ were similar between $c d 14-/-$ and WT cells, consistent with the impact or lack of impact of Kgp on the response to activation with these factors. 


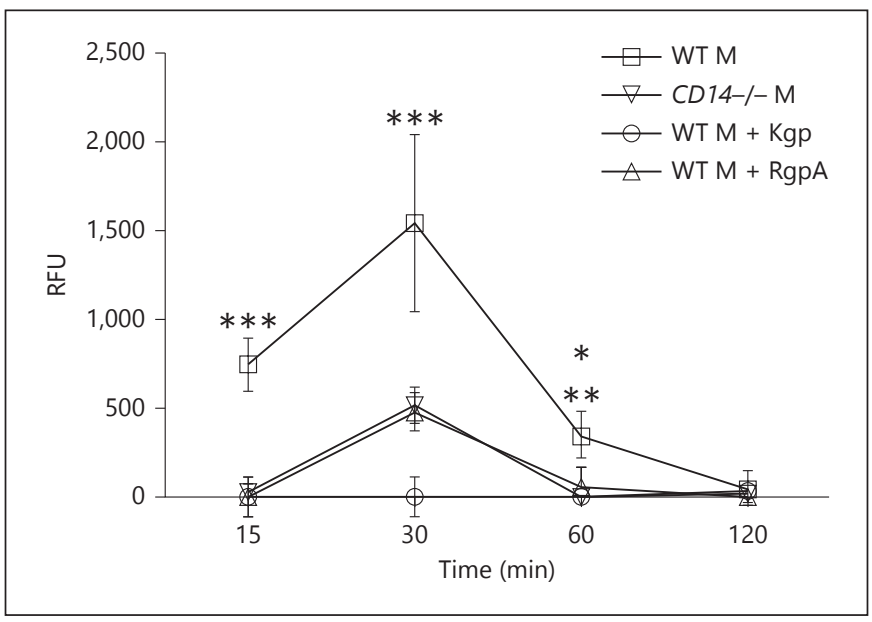

Fig. 4. Phagocytosis of $P$. gingivalis by macrophages is reduced following pretreatment with purified gingipains. Kgp- or RgpAtreated macrophages $(\mathrm{M})$ were incubated with FITC-labeled $P$. gingivalis for 15-120 min. Extracellular fluorescence was quenched with trypan blue $(1.25 \mathrm{mg} / \mathrm{ml})$ prior to fluorescence measurement. Values represent the means of relative fluorescence units (RFU) \pm SD of one representative experiment out of three repeats with similar results. Purified Kgp and RgpA significantly reduced phagocytosis at 15 and $30 \mathrm{~min}\left({ }^{* * *} \mathrm{p}<0.001\right)$ and at $60 \mathrm{~min}\left({ }^{* *} \mathrm{p}<0.01\right.$ for Kgp; * $\mathrm{p}<0.05$ for Rgp). The level of phagocytosis by $c d 14-/-$ cells was similar to RgpA-pretreated WT cells at all time points.

These results suggest that Kgp mediates reduced CD14 expression that correlates with a reduced macrophage response to $P$. gingivalis infection.

\section{Macrophage Pretreatment with Gingipains Reduces \\ Phagocytosis of P. gingivalis}

We next asked whether diminished CD14, in the presence of unchanged levels of TLR2 and TLR4, impacts upon phagocytosis of $P$. gingivalis. Untreated cells phagocytosed $P$. gingivalis in a time-dependent manner, reaching peak activity at $30 \mathrm{~min}$ (fig. 4). Pretreatment with purified Kgp and RgpA significantly reduced phagocytosis at 15 and $30 \mathrm{~min}(\mathrm{p}<0.001)$ and at $60 \mathrm{~min}(\mathrm{p}<0.01$ for Kgp; $\mathrm{p}<0.05$ for Rgp; fig. 4). Phagocytosis of $P$. gingivalis by $c d 14-/-$ macrophages was similar to that of WT cells pretreated with RgpA, suggesting that Kgp affects additional receptors involved in phagocytosis.

\section{Discussion}

Our results show that the purified gingipains RgpA and Kgp, as well as supernatants from WT $P$. gingivalis $\mathrm{W} 83$, and the single mutants rgp $\mathrm{A}-$ and $\mathrm{kgp}-$, cleave sur- face-expressed CD14 in a concentration- and a time-dependent manner. Reduced CD14 expression was dependent on the presence of a hemagglutinin/adhesion domain since the Arg-gingipain RgpB, which contains a nearly identical catalytic domain to RgpA but lacks the adhesion domain, was not active. The hemagglutinin/adhesion site binds fibrinogen, fibronectin and laminin to interact with cell membrane phospholipids thus increasing the protease affinity to its substrate [23]. Furthermore, the reduced CD14 expression was significantly more sensitive to Lys-gingipain activity in comparison to Arg-gingipain activity. Importantly, Kgp and RgpA reduced CD14 expression even at $0.1 \mu \mathrm{M}$, a concentration that is well within the recently reported gingipain concentrations found in gingival crevicular fluid (GCF; $\leq 1.5 \mu \mathrm{M}$ ) [24]. Though the GCF levels are only directly relevant to the CD14-positive cells that have migrated through the tissue to the gingival sulcus [25], the levels measured in the GCF provide some indication of what can be expected in vivo. In addition, CD14 in vivo surface expression was reported to be greater in clinically healthy gingival pockets than in periodontitis patients, suggesting that greater CD14 surface expression correlates with periodontal health [26].

Our findings regarding CD14 expression following macrophage exposure to gingipains are consistent with results from previous studies on human monocytes and macrophages [17, 27]. Both human monocyte CD14 and murine macrophage CD14 are cleaved more efficiently by gingipains containing the hemagglutinin/adhesion domain. However, in contrast to human monocyte CD14, murine macrophage CD14 expression is more rapidly and efficiently reduced by Lys-gingipain than Arg-gingipain. This may be explained by the presence of 4 more arginine residues in human CD14 in comparison to the murine protein [28]. Analysis of solvent accessible lysine and arginine residues in the murine CD14 crystal structure did not provide an explanation for the greater influence of Lys-gingipain relative to Arg-gingipain over CD14 expression since there are 9 solvent-exposed residues of each amino acid (http://www.rcsb.org/pdb/explore/remediatedSequence.do? structureId $=1 \mathrm{WWL}$ ).

Of significance, previous reports have shown TLR2, rather than TLR4, to be critical for the host response to infection with $P$. gingivalis. In the absence of TLR2, the in vitro and in vivo cytokine response to $P$. gingivalis is diminished $[15,16]$. In addition, TLR2 activation is also central to the evasion strategy of $P$. gingivalis since TLR2deficient mice are able to rapidly clear infection, as are TLR2-deficient macrophages in vitro [29]. Therefore, it 
was critical to determine the effect of gingipains on this pattern recognition receptor since it was not reported previously. In contrast to $\mathrm{CD} 14$, gingipains did not reduce detectable surface expression of TLR2 or TLR4, and their expression was consistently unaffected by any of the gingipains in any of the conditions tested. In retrospect, the fact that TLR2 expression remains unchanged is not surprising, given that multiple reports have shown that TLR2, and not TLR4, is critical for the responsiveness of cells to $P$. gingivalis LPS [30] and to the whole bacteria [16].

CD14 is a coreceptor for multiple TLRs [11, 12]; however, its relative importance for the cellular response depends on the particular TLR ligand. We found that reduced CD14 expression mediated by Kgp did not affect the macrophage response to the TLR2/TLR1 ligand $\mathrm{Pam}_{3} \mathrm{CSK}_{4}$, but the response to infection with live $P$. gingivalis and E. coli LPS was significantly reduced. Furthermore, the response of CD14-deficient cells to infection with $P$. gingivalis, but not to challenge with $\mathrm{Pam}_{3} \mathrm{CSK}_{4}$, was also reduced compared to WT macrophages. Although some studies have found CD14 to physically bind triacylated lipopeptides such as $\mathrm{Pam}_{3} \mathrm{CSK}_{4}$ and induce physical proximity to the TLR2/TLR1 complex [31], our results are consistent with other reports that CD14 is dispensable for the cellular response to $\mathrm{Pam}_{3} \mathrm{CSK}_{4}[32,33]$. The macrophage response to HKS $P$. gingivalis did not require CD14, presumably because multiple TLR and non-TLR ligands are exposed by heat and sonication. Importantly, pre-exposure to both Arg- or Lys-gingipains resulted in reduced phagocytosis of $P$. gingivalis by macrophages. Phagocytosis ability of $c d 14-/-$ macrophages resembled that of WT macrophages pretreated with
RgpA, pointing to a possible role of CD14 in bacterial elimination by macrophages. This emphasizes the possibility that the bacteria evade elimination by the host through reducing CD14 expression on macrophages, which in turn may result in reduced cytokine responses and a lower ability to phagocytose bacteria.

Taken together, our findings suggest that gingipains selectively influence the surface expression of innate immune receptors important for the recognition of live P. gingivalis, leaving surface immune receptors linked to bacterial persistence intact. The accumulation of gingipains [34] in inflammatory periodontal lesions can reduce innate receptor expression and thereby alter the responsiveness of macrophages ahead of their encounter with the bacteria. Further studies will examine whether $P$. gingivalis signaling via TLR2 in the absence of CD14 leads to improved bacterial persistence and greater pathogenicity.

\section{Acknowledgments}

This research was supported, in part, by the Israel Science Foundation (grant No. 1396/12*) to G.N. and the Internal Fund of the Hadassah Medical Organization to A.W. J.P. acknowledges support from the National Institutes of Health, USA (grants DE 09761 and DE022597), the National Science Center, Poland (2011/01/B/ NZ6/00268), the Foundation for Polish Science (TEAM project DPS/424-329/10), the Ministry of Science and Higher Education, Poland (2137/7. PR-EU/2011/2), and the European Community (FP7-HEALTH-2010-261460 'Gums\&Joints', FP7-HEALTH-2012INNOVATION-306029 'TRIGGER', and PITN-GA-2011-290246, 'RAPID'). The Faculty of Biochemistry, Biophysics and Biotechnology of the Jagiellonian University is a beneficiary of structural funds from the European Union [POIG.02.01.00-12-064/08].

\section{References}

1 Lamont RJ, Jenkinson HF: Life below the gum line: pathogenic mechanisms of Porphyromonas gingivalis. Microbiol Mol Biol Rev 1998;62:1244-1263.

2 Holt SC, Kesavalu L, Walker S, Genco CA: Virulence factors of Porphyromonas gingivalis. Periodontol 2000 1999;20:168-238.

3 Imamura T: The role of gingipains in the pathogenesis of periodontal disease. J Periodontol 2003;74:111-118.

4 Andrian E, Grenier D, Rouabhia M: Porphyromonas gingivalis-epithelial cell interactions in periodontitis. J Dent Res 2006;85: 392-403.

5 Mezyk-Kopec R, Bzowska M, Potempa J, Jura $\mathrm{N}$, Sroka A, Black RA, Bereta J: Inactivation of membrane tumor necrosis factor alpha by gingipains from Porphyromonas gingivalis. Infect Immun 2005;73:1506-1514.
6 Vincents B, Guentsch A, Kostolowska D, von Pawel-Rammingen U, Eick S, Potempa J, Abrahamson M: Cleavage of IgG1 and IgG3 by gingipain $\mathrm{K}$ from Porphyromonas gingivalis may compromise host defense in progressive periodontitis. FASEB J 2011;25:37413750.

7 Means TK, Golenbock DT, Fenton MJ: Structure and function of Toll-like receptor proteins. Life Sci 2000;68:241-258.

8 Poltorak A, He X, Smirnova I, Liu MY, Van Huffel C, Du X, Birdwell D, Alejos E, Silva M, Galanos C, Freudenberg M, Ricciardi-Castagnoli P, Layton B, Beutler B: Defective LPS signaling in $\mathrm{C} 3 \mathrm{H} / \mathrm{HeJ}$ and $\mathrm{C} 57 \mathrm{BL} / 10 \mathrm{ScC}$ mice: mutations in Tlr4 gene. Science 1998;282: 2085-2088.

-9 Aliprantis AO, Yang RB, Mark MR, Suggett S, Devaux B, Radolf JD, Klimpel GR, Godowski
P, Zychlinsky A: Cell activation and apoptosis by bacterial lipoproteins through Toll-like receptor-2. Science 1999;285:736-739.

10 Bauer S, Kirschning CJ, Hacker H, Redecke V, Hausmann S, Akira S, Wagner H, Lipford GB: Human TLR9 confers responsiveness to bacterial DNA via species-specific CpG motif recognition. Proc Natl Acad Sci USA 2001;98: 9237-9242.

-11 Baumann CL, Aspalter IM, Sharif O, Pichlmair A, Bluml S, Grebien F, Bruckner M, Pasierbek P, Aumayr K, Planyavsky M, Bennett KL, Colinge J, Knapp S, Superti-Furga G: CD14 is a coreceptor of Toll-like receptors 7 and 9. J Exp Med 2010;207:2689-2701.

12 Fitzgerald KA, Rowe DC, Golenbock DT: Endotoxin recognition and signal transduction by the TLR4/MD2-complex. Microbes Infect 2004;6:1361-1367. 
13 Simmons DP, Canaday DH, Liu Y, Li Q, Huang A, Boom WH, Harding CV: Mycobacterium tuberculosis and TLR2 agonists inhibit induction of type I IFN and class I MHC antigen cross processing by TLR9. J Immunol 2010;185:2405-2415.

14 Montminy SW, Khan N, McGrath S, Walkowicz MJ, Sharp F, Conlon JE, Fukase K, Kusumoto S, Sweet C, Miyake K, Akira S, Cotter RJ, Goguen JD, Lien E: Virulence factors of Yersinia pestis are overcome by a strong lipopolysaccharide response. Nat Immunol 2006;7: 1066-1073.

15 Hajishengallis G, Ratti P, Harokopakis E: Peptide mapping of bacterial fimbrial epitopes interacting with pattern recognition receptors. J Biol Chem 2005;280:3890238913.

16 Burns E, Bachrach G, Shapira L, Nussbaum G: Cutting edge: TLR2 is required for the innate response to Porphyromonas gingivalis: activation leads to bacterial persistence and TLR2 deficiency attenuates induced alveolar bone resorption. J Immunol 2006;177:8296-8300.

-17 Sugawara S, Nemoto E, Tada H, Miyake K, Imamura T, Takada H: Proteolysis of human monocyte CD14 by cysteine proteinases (gingipains) from Porphyromonas gingivalis leading to lipopolysaccharide hyporesponsiveness. J Immunol 2000;165: 411-418.

18 Leijh PC, van Zwet TL, ter Kuile MN, van Furth R: Effect of thioglycolate on phagocytic and microbicidal activities of peritoneal macrophages. Infect Immun 1984;46: 448-452.

19 Potempa J, Mikolajczyk-Pawlinska J, Brassell D, Nelson D, Thogersen IB, Enghild JJ, Travis J: Comparative properties of two cysteine proteinases (gingipains $\mathrm{R}$ ), the products of two related but individual genes of Porphyromonas gingivalis. J Biol Chem 1998;273: 21648-21657.
20 Liu X, Sroka A, Potempa J, Genco CA: Coordinate expression of the Porphyromonas gingivalis lysine-specific gingipain proteinase, Kgp, arginine-specific gingipain proteinase, RgpA, and the heme/hemoglobin receptor, HmuR. Biol Chem 2004;385:1049-1057.

21 Chen Z, Potempa J, Polanowski A, Wikstrom $\mathrm{M}$, Travis J: Purification and characterization of a $50-\mathrm{kDa}$ cysteine proteinase (gingipain) from Porphyromonas gingivalis. J Biol Chem 1992;267:18896-18901.

22 Pike R, McGraw W, Potempa J, Travis J: Lysine- and arginine-specific proteinases from Porphyromonas gingivalis. Isolation, characterization, and evidence for the existence of complexes with hemagglutinins. J Biol Chem 1994;269:406-411.

-23 Tada H, Sugawara S, Nemoto E, Takahashi N, Imamura T, Potempa J, Travis J, Shimauchi $\mathrm{H}$, Takada H: Proteolysis of CD14 on human gingival fibroblasts by arginine-specific cysteine proteinases from Porphyromonas gingivalis leading to down-regulation of lipopolysaccharide-induced interleukin-8 production. Infect Immun 2002;70:3304-3307.

24 Guentsch A, Kramesberger M, Sroka A, Pfister W, Potempa J, Eick S: Comparison of gingival crevicular fluid sampling methods in patients with severe chronic periodontitis. J Periodontol 2011;82:1051-1060.

25 Kornman KS, Page RC, Tonetti MS: The host response to the microbial challenge in periodontitis: assembling the players. Periodontol 2000 1997; 14:33-53.

26 Jin L, Ren L, Leung WK, Darveau RP: The in vivo expression of membrane-bound CD14 in periodontal health and disease. J Periodontol 2004;75:578-585.

27 Duncan L, Yoshioka M, Chandad F, Grenier D: Loss of lipopolysaccharide receptor CD14 from the surface of human macrophage-like cells mediated by Porphyromonas gingivalis outer membrane vesicles. Microb Pathog 2004;36:319-325.
28 Setoguchi M, Nasu N, Yoshida S, Higuchi Y, Akizuki S, Yamamoto S: Mouse and human CD14 (myeloid cell-specific leucine-rich glycoprotein) primary structure deduced from cDNA clones. Biochim Biophys Acta 1989; 1008:213-222.

29 Liang S, Krauss JL, Domon H, McIntosh ML, Hosur KB, Qu H, Li F, Tzekou A, Lambris JD, Hajishengallis G: The C5a receptor impairs IL-12-dependent clearance of Porphyromonas gingivalis and is required for induction of periodontal bone loss. J Immunol 2011;186: 869-877.

30 Darveau RP, Pham TT, Lemley K, Reife RA, Bainbridge BW, Coats SR, Howald WN, Way SS, Hajjar AM: Porphyromonas gingivalis lipopolysaccharide contains multiple lipid A species that functionally interact with both Toll-like receptors 2 and 4. Infect Immun 2004;72:5041-5051.

31 Manukyan M, Triantafilou K, Triantafilou M, Mackie A, Nilsen N, Espevik T, Wiesmuller $\mathrm{KH}$, Ulmer AJ, Heine H: Binding of lipopeptide to CD14 induces physical proximity of CD14, TLR2 and TLR1. Eur J Immunol 2005; 35:911-921.

32 Court N, Rose S, Bourigault ML, Front S, Martin OR, Dowling JK, Kenny EF, O’Neill L, Erard F, Quesniaux VF: Mycobacterial PIMs inhibit host inflammatory responses through CD14-dependent and CD14-independent mechanisms. PLoS One 2011;6:e24631.

33 Jiang Z, Georgel P, Du X, Shamel L, Sovath S, Mudd S, Huber M, Kalis C, Keck S, Galanos C, Freudenberg M, Beutler B: CD14 is required for MyD88-independent LPS signaling. Nat Immunol 2005;6:565-570.

-34 Dias IH, Marshall L, Lambert PA, Chapple IL, Matthews JB, Griffiths HR: Gingipains from Porphyromonas gingivalis increase the chemotactic and respiratory burst-priming properties of the 77-amino-acid interleukin-8 variant. Infect Immun 2008;76:317-323.
Selective Reduction in CD14 Expression by $P$. gingivalis Gingipains
J Innate Immun 2015;7:127-135 DOI: $10.1159 / 000365970$ 